\title{
Weibull Distribution Parameter Estimation of the Algorithm Based on the Improved Flies
}

\author{
Ji Xiu1,2,a, Wang Hui1,2,b, ZHAO Chuan-qi1,2,c , Si Yuan1,2,a \\ 1College of Electrical and Information Engineering, Changchun \\ Institute of Technology,Changchun Changchun China,130012 \\ 2Public Technology Research and Development Center of Distribution \\ Equipment Automation Industry in Jilin Province, Changchun \\ China,130012 \\ ajixiu523@163.com,b254425251@qq.com,c706659188@qq.com
}

\begin{abstract}
Based on weibull distribution model parameters of maximum likelihood estimation process trival, is obtained by using the Newton method and adopting the particle swarm optimization algorithm is easy to fall into premature and program variables is more complicated problems in the implementation, put optimization algorithm is introduced into maximum likelihood method, and to improve the conventional fruit flies algorithm is introduced into step length factor, put forward the optimization algorithm based on improved fruit flies weibull distribution parameters estimation method, and compared with Newton's method and were compared with normal flies algorithm of particle swarm optimization (pso), the feasibility and effectiveness of the method is verified by examples.

Keywords: Weibull distribution; Parameter estimation; Maximum likelihood estimation; Fruit flies optimization algorithm

\section{Introduction}

Weibull distribution model is one of the reliability model, has been used in engineering reliability analysis, mechanical equipment failure life data analysis and survival analysis of medicine in many fields, such as different levels of applications[1]are obtained. This paper introduced optimization theory, improve flies to join step factor in the process of weibull distribution parameter estimation, the first to establish the likelihood function of weibull distribution, and then flies optimization algorithm is adopted to define the function extreme value to determine the estimate of parameters[2], and with the Newton method and particle swarm optimization (pso) algorithm and conventional flies algorithm simulated the comparative analysis, the method is proven to be overcome in the process of weibull distribution parameter estimation earliness and convergence speed advantages [3][4].
\end{abstract}




\section{Weibull distribution parameters of maximum likelihood estimate}

The two parameter weibull distribution density function and cumulative distribution functions are:

$$
\begin{array}{r}
f(t)=\frac{\beta}{\eta}\left(\frac{t}{\eta}\right)^{\beta-1} \exp \left[-\left(\frac{t}{\eta}\right)^{\beta}\right] \\
F(t)=1-\exp \left[-\left(\frac{t}{\eta}\right)^{\beta}\right]
\end{array}
$$

Assuming overall obeys two parameter weibull distribution, the probability density function is:

$$
f(t ; \beta, \eta)=\frac{\beta}{\eta}\left(\frac{t}{\eta}\right)^{\beta-1} \exp \left[-\left(\frac{t}{\eta}\right)^{\beta}\right]
$$

Set toT1,T2,...,Tn separate $\mathrm{n}$ from the overall sample, sample observation value is $\mathrm{t} 1, \mathrm{t} 2, \ldots, \mathrm{tn}$, the $\mathrm{t} 1, \mathrm{t} 2, \ldots, \mathrm{tn}$, likelihood function is:

$$
L(\beta, \eta)=\prod_{i=1}^{n} f\left(t_{i} ; \beta, \eta\right)=\prod_{i=1}^{n} \frac{\beta}{\eta}\left(\frac{t_{i}}{\eta}\right)^{\beta-1} \exp \left[-\left(\frac{t_{i}}{\eta}\right)^{\beta}\right]
$$

Weibull distribution parameters of maximum likelihood estimation is in the range of $(\beta, \eta)$ possible values selection $L(\beta, \eta)$ makes the biggest parameter $(\hat{\beta}, \hat{\eta})$ values, as the estimate of $(\beta, \eta)$ the type (4) the exponential, are:

$$
l(\beta, \eta)=\ln L(\beta, \eta)=n \ln \frac{\beta}{\eta}+\sum_{i=1}^{n}\left(-\left(\frac{t_{i}}{\eta}\right)^{\beta}+(\beta-1) \ln \left(\frac{t_{i}}{\eta}\right)\right)
$$

Therefore, parameters of $(\beta, \eta)$ maximum likelihood estimation problem into the problem of the $l(\beta, \eta)$ maximum, the partial derivatives $l(\beta, \eta)$, and establish a system of equations[5]:

$$
\left\{\begin{array}{c}
\frac{\partial l}{\partial \beta}=\frac{n}{\beta}+\sum_{i=1}^{n}\left(-\left(\frac{t_{i}}{\eta}\right)^{\beta} \ln \left(\frac{t_{i}}{\eta}\right)+\ln \left(\frac{t_{i}}{\eta}\right)\right)=0 \\
\frac{\partial l}{\partial \eta}=-\frac{n \beta}{\eta}+\frac{\beta}{\eta} \sum_{i=1}^{n}\left(\frac{t_{i}}{\eta}\right)^{\beta}=0
\end{array}\right.
$$


For the equations using the Newton iteration method to solve, estimates $(\hat{\beta}, \hat{\eta})$ can be obtained.

\section{Weibull distribution based on the theory of the fruit flies to optimize parameters of maximum likelihood estimate}

Fruit Fly Optimization Algorithm is a kind of simulation fruit flies group foraging behavior by the sense of smell and vision of global optimization method. First randomly initialize the flies group position, in the process of iteration, giving fruit flies and calculate the individual random flight direction and distance taste concentration Determine concentration value $S$ and taste Smell=function(S) One $S$ function is taste concentration decision function for drosophila individual position, the reciprocal of the distance to the origin, and the optimal solution by comparing group and retain the best global optimal solution concentration and its location, until the maximum number of iterations.

Due to the system of equations (6) is a set of transcendental equations, the solving process is relatively complex, so will fly optimization algorithm of weibull distribution parameters of maximum likelihood estimation, Select two parameters weibull distribution of negative logarithmic likelihood function $-l(\beta, \eta)$ as the taste of fruit flies optimization algorithm concentration decision function directly to find the minimum value, and the overall method process is as follows:

(1) The initialization flies group number sizepop, the number of iterations maxgen and the initial position:

$\left\{\begin{array}{l}X_{-} \text {axis }_{k}^{i} \\ Y_{\text {_axis }}{ }_{k}\end{array}\right.$

(2) Give fruit flies the individual random flight direction and distance:

$\left\{\begin{aligned} X_{k}^{i} & =X_{-}{ }_{a x i s}^{i}+\text { rand } \\ Y_{k}^{i} & =Y_{-}{ }_{\text {axis }}^{i}+\text { rand }\end{aligned}\right.$

Which rand is between the random number between[-1,1], on behalf of individual random flight direction and distance.

(3) Calculate all individual flies the current position and to determine the distance Dist between the origin, taste concentration value $S$ and taste concentration Smell:

$$
\left\{\begin{array}{c}
\text { Dist }_{k}^{i}=\sqrt{\left[\left(X_{k}^{i}\right)^{2}+\left(Y_{k}^{i}\right)^{2}\right]} \\
S_{k}^{i}=1 / \operatorname{Dist}_{k}^{i} \\
\text { Smell }_{k}=\text { function }\left(S_{k}^{i}\right)=-l\left(S_{k}^{1}, S_{k}^{2}\right)
\end{array}\right.
$$

(4) The corresponding flies to the groups minimum density and taste of 
the individual position:

[bestSmell, bestIndex $]=\min \left(\right.$ Smell $\left._{k}\right)$

(5) Retain the best taste density and individual location, location update flies groups:

$$
\left\{\begin{array}{c}
\text { Smellbest }=\text { bestSmell } \\
X_{\text {_axis }}{ }_{k+1}^{i}=X_{\text {bestIndex }}^{i} \\
Y_{\text {_axis }}{ }_{k+1}^{i}=Y_{\text {bestIndex }}^{i}
\end{array}\right.
$$

(6) Fruit flies iterative optimization. Repeat steps (2) - (4), and to determine whether a taste concentration is less than the previous iteration flavor concentration, if is executed (5).

To overcome the problem of slow convergence speed, improve the convergence of fruit flies, on the basis of the formula (8) add step length factor to improve fruit flies algorithm, formula is as follows:

$$
\left\{\begin{array}{c}
X_{k}^{i}=X_{-} \text {axis }{ }_{k}^{i}+h_{k} * \text { rand } \\
Y_{k}^{i}=Y_{-} \text {axis }{ }_{k}^{i}+h_{k} * \text { rand }
\end{array}\right.
$$

For the $0<h_{k} \leq 1$ step length factor, specific as follows:

$$
\begin{aligned}
& h_{k}=\delta^{*} h_{k-1} \\
& \left\{\begin{array}{c}
\delta=1, \quad \text { bestSmell }<\text { Smellbest } \\
\delta=0.9 \quad \text { Smellbest } \geq \text { bestSmell }
\end{array}\right.
\end{aligned}
$$

The initialization step for $h_{0}=1$ diminishing $\delta$ step length factor.

\section{Case analysis}

A type in literature [6] is adopted to the fault of diesel engine cylinder liner range sample data to parameter estimation as the experimental data, the order of the sample data statistics as shown in table 1,.Respectively using Newton method and particle swarm optimization (pso) algorithm, algorithm for conventional fruit flies and flies algorithm of two parameter weibull distribution parameter estimation, and the experimental results are compared.

Table 1 Sample data 


\begin{tabular}{|c|c|c|c|c|c|c|c|}
\hline $\begin{array}{l}\text { Serial } \\
\text { number }\end{array}$ & $\begin{array}{l}\text { fault } \\
\text { mileage } \\
\backslash \quad 103 \\
\text { kilometers }\end{array}$ & $\begin{array}{l}\text { Serial } \\
\text { number }\end{array}$ & $\begin{array}{l}\text { fault } \\
\text { mileage } \\
\backslash \quad 103 \\
\text { kilometers }\end{array}$ & $\begin{array}{c}\text { Serial } \\
\text { number }\end{array}$ & $\begin{array}{l}\text { fault } \\
\text { mileage } \\
\backslash \quad 103 \\
\text { kilometers }\end{array}$ & $\begin{array}{l}\text { Serial } \\
\text { number }\end{array}$ & $\begin{array}{l}\text { fault } \\
\text { mileage } \\
\backslash \quad 103 \\
\text { kilometers }\end{array}$ \\
\hline 1 & 6.4 & 26 & 23 & 14 & 18 & 39 & 32.1 \\
\hline 2 & 7.99 & 27 & 24 & 15 & 18.43 & 40 & 34.9 \\
\hline 3 & 8.6 & 28 & 25 & 16 & 18.93 & 41 & 36 \\
\hline 4 & 10 & 29 & 26.06 & 17 & 19 & 42 & 36.52 \\
\hline 5 & 10.4 & 30 & 26.5 & 18 & 19.8 & 43 & 37 \\
\hline 6 & 11.8 & 31 & 27 & 19 & 20 & 44 & 37.05 \\
\hline 7 & 12.3 & 32 & 27.8 & 20 & 21 & 45 & 37.2 \\
\hline 8 & 13 & 33 & 27.84 & 21 & 21.19 & 46 & 42 \\
\hline 9 & 15 & 34 & 28.3 & 22 & 21.3 & 47 & 42.16 \\
\hline 10 & 16.5 & 35 & 29.1 & 23 & 21.5 & 48 & 43.2 \\
\hline 11 & 16.85 & 36 & 29.72 & 24 & 22 & 49 & 43.38 \\
\hline 12 & 16.9 & 37 & 29.8 & 25 & 22.12 & 50 & 45 \\
\hline 13 & 17.6 & 38 & 31 & & & & \\
\hline
\end{tabular}

swarm optimization (pso) algorithm, take learning factor $c_{1}=c_{2}=2.0$, The largest number of iterations maxgen $=500$,Population size sizepop=30, Inertia coefficient $\omega$,using the type (14) update:

$$
\omega=\left(\omega_{\text {max }} / \omega_{\text {min }}\right)^{\frac{1}{1+10 i / \text { maxgen }}}
$$

Which $i$ is the number of iterations, $\omega_{\max }=0.9, \omega_{\min }=0.4$ Fruit flies algorithm,Maximum number of iterations maxgen=500,Population size sizepop=30,Group the initial position $[-1,1]$.

Of the above four methods respectively under the environment of matlab simulation, get the best results, figure 1 shows the searching process is iterative algorithm, the final result of table 2 lists the comparison. 


\begin{tabular}{cccc}
\hline & $\begin{array}{l}\text { Particle swarm } \\
\text { optimization } \\
\text { (pso) }\end{array}$ & $\begin{array}{l}\text { Fruit flies } \\
\text { algorithm }\end{array}$ & $\begin{array}{l}\text { Algorithm to } \\
\text { improve fruit } \\
\text { flies }\end{array}$ \\
\hline$\beta$ & 2.6407 & 2.6468 & 2.6994 \\
$\eta$ & 27.7053 & 27.6983 & 27.4841 \\
$\min (-l(\beta, \eta))$ & 185.2938 & 185.2935 & 185.3312 \\
$\begin{array}{c}\text { Computing time } \\
(\mathrm{s})\end{array}$ & 1.6236 & 1.1832 & 1.1658 \\
\hline
\end{tabular}

Table 2 Particle swarm optimization (pso) algorithm and Fruit flies iteration of the algorithm

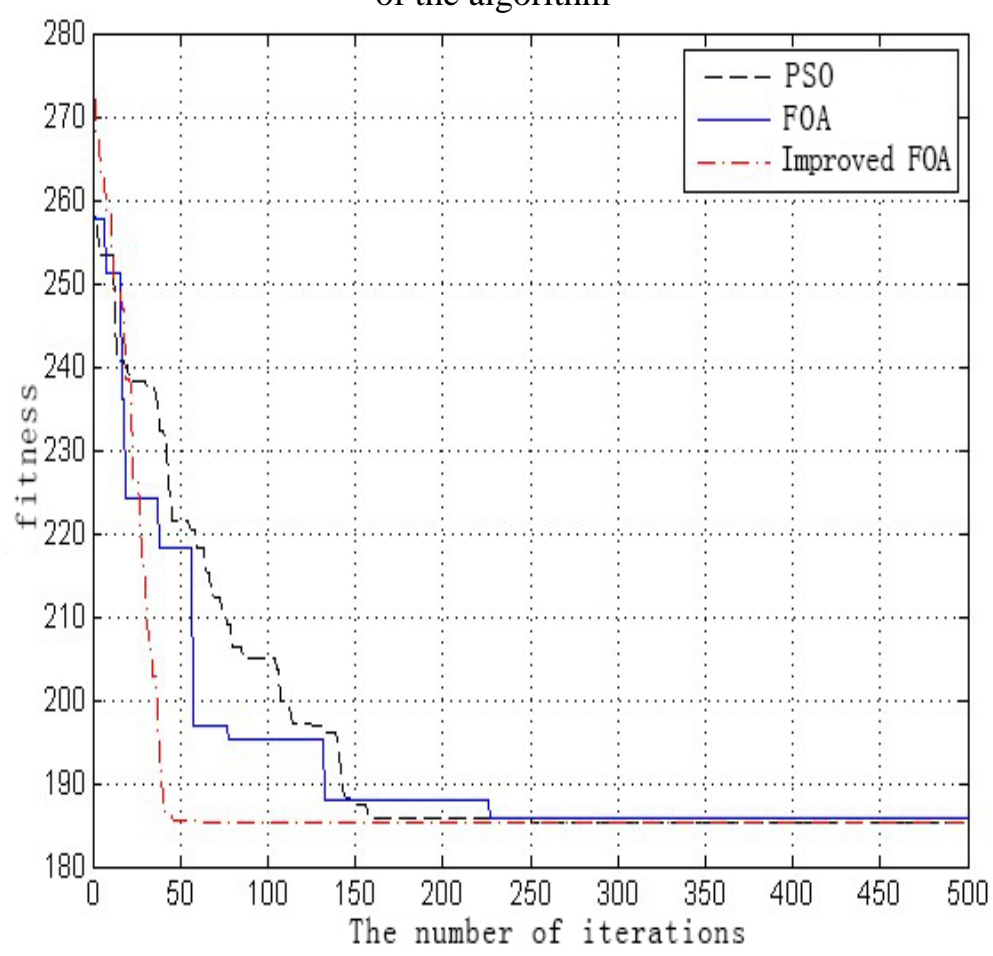

Figure 1 Sample data fitting result contrast

As can be seen from table 2, the improvement of fruit flies algorithm can more accurately find the minimum value and calculation time consuming is better than that of particle swarm algorithm; Can be seen from figure 1, the improvement of 
fruit flies algorithm with faster convergence speed and convergence, the smaller the number of iterations at the same time flies algorithm in the process of program implementation required variable is less than the particle swarm algorithm, reduce the difficulty in application.

\section{Conclusion}

This article in the drosophila optimization algorithm was improved, join step factor is applied to the two parameter weibull distribution parameters of maximum likelihood estimation and simulation verification. Based on the sample data, using particle swarm optimization (pso), conventional flies algorithm and improvement of fruit flies, the two parameters weibull distribution parameters are estimated and the results of comparative analysis. Experimental results show that the improved flies optimization algorithm in two parameter weibull distribution parameters of maximum likelihood estimation of overcome the premature problem of conventional flies algorithm, convergence rate is better than fruit flies algorithm at the same time, with high precision, low computing time consuming and the advantage of fast convergence speed.

\section{Acknowledgement}

In this paper, the research was sponsored by Jilin Province Development and Reform Commission research projects (20131188-31), Changchun technology bureau Major science and technology research projects(No.2013266) and Jilin Province Science and Technology Development Program (No20130206049G X)

\section{References}

[1] Chun-ping li. is completely samples under weibull distribution parameter of the weighted least squares estimation [J]. Journal of xiaogan college journal, 2013 (6) : 83-8.

[2] yan-qing wen, bao-liang liu. Weibull distribution under the condition of complete data of the parameter estimation [J]. Journal of shanxi datong university, 2009, 25 (4) : 17-19.

[3] Xue Yuxia, ShenGuiXiang ying-zhi zhang, jia Asia. Reliability distribution model based on particle swarm optimization theory of the maximum likelihood parameter estimation method [J]. Journal of jilin university (engineering science), 2009, 33 (S1) 6:219-221.

[4] Dong Sheng, Korea, tao mountains, FanDuiQiu. Weibull distribution parameters of particle swarm optimization (pso) algorithm to estimate [J]. Chinese journal of ocean university (natural science edition), 2012, and (6) : 120-125. 
[5] Xu Wei, wei-ming hu, his. Based on the two parameter weibull distribution equipment reliability prediction research [J]. Journal of China construction machinery, 2013, 11 (02) : 112-116.

[6] LingDan. Weibull distribution model and its application in the mechanical reliability research [D]. Chengdu: university of electronic science and technology, 2011. 